\title{
Nonlinear Plasmonic Sensing with Nanographene
}

\author{
Renwen Yu, ${ }^{1}$ Joel D. Cox, ${ }^{1}$ and F. Javier García de Abajo ${ }^{1,2, *}$ \\ ${ }^{1}$ ICFO-Institut de Ciencies Fotoniques, The Barcelona Institute of Science and Technology, \\ 08860 Castelldefels (Barcelona), Spain \\ ${ }^{2}$ ICREA-Institució Catalana de Recerca i Estudis Avançats, Passeig Lluís Companys 23, \\ 08010 Barcelona, Spain
}

(Received 12 May 2016; published 16 September 2016)

\begin{abstract}
Plasmons provide excellent sensitivity to detect analyte molecules through their strong interaction with the dielectric environment. Plasmonic sensors based on noble metals are, however, limited by the spectral broadening of these excitations. Here we identify a new mechanism that reveals the presence of individual molecules through the radical changes that they produce in the plasmons of graphene nanoislands. An elementary charge or a weak permanent dipole carried by the molecule are shown to be sufficient to trigger observable modifications in the linear absorption spectra and the nonlinear response of the nanoislands. In particular, a strong second-harmonic signal, forbidden by symmetry in the unexposed graphene nanostructure, emerges due to a redistribution of conduction electrons produced by interaction with the molecule. These results pave the way toward ultrasensitive nonlinear detection of dipolar molecules and molecular radicals that is made possible by the extraordinary optoelectronic properties of graphene.
\end{abstract}

DOI: 10.1103/PhysRevLett.117.123904

Localized surface plasmons (LSPs) have attracted considerable attention in the nanophotonics community due to their pivotal role in optical sensing applications such as antibody-antigen [1-3], gas [4,5], and $p \mathrm{H}[6,7]$ sensors. These excitations also enable the detection and chemical identification of single molecules through their enhancement of molecule-specific Raman scattering intensities [8-13]. LSPs are routinely observed in noble metal nanostructures, appearing as pronounced spectral features in their optical absorption and scattering spectra. Plasmonbased sensing heavily relies on the ability of these collective modes to confine and strongly amplify the optical near field. These properties are equally responsible for the large nonlinear optical response observed in metal nanoparticles [14-19], which has inspired alternative mechanisms for nonlinear plasmonic sensing. For instance, the aggregation of gold nanoparticles caused by targeted heavy metal ions [20], Escherichia coli bacteria [21], or Alzheimer's disease biomarkers [22] can be detected through an increase in second-harmonic generation (SHG). Additionally, third-harmonic generation has been recently claimed to offer large sensitivity to the dielectric environment compared to the linear response [23].

Doped graphene is widely recognized as a promising material platform for plasmonics, capable of supporting electrically tunable plasmons with higher quality factors and spatial confinement than those of metal nanoparticles [24-37]. Moreover, tunable graphene plasmons, so far observed at midinfrared (IR) and THz frequencies, provide the strong near-electric-field confinement needed for sensing [38-41]. In particular, graphene plasmons have been demonstrated to reveal vibrational fingerprints of biomolecules [38]. Additionally, the anharmonic electron motion associated with the Dirac cones of this material $[42,43]$ gives rise to an extraordinary nonlinear response in extended samples [44-46]. LSPs in graphene nanoislands have been predicted to produce unprecedentedly high harmonic generation and wave mixing $[47,48]$, indicating their strong capability for nonlinear optical sensing.

In this Letter, we show through atomistic quantummechanical simulations that both the linear and nonlinear optical response of graphene nanoislands can be dramatically altered by the presence of a single neighboring molecule that carries either an elementary charge or a permanent dipole of only a few Debye. As a proofof-principle demonstration, we focus on small graphene nanohexagons (GNHs), similar to those produced with high quality through chemical synthesis $[49,50]$. Our calculations indicate that the presence of an analyte can significantly modify the distribution of conduction electrons in GNHs that are a few nanometers in lateral size [51], leading to new plasmonic features in their linear optical response. This interaction may also break the nanoisland inversion symmetry, thus enabling plasmon-enhanced SHG [52]. Analyte-induced plasmon features in the absorption spectra and increased SHG intensities occur even for initially undoped GNHs, suggesting that nanographenes can serve as a tunable and efficient platform for detecting charge- or dipole-carrying molecules.

The small size of the GNHs under consideration demands an atomistic level of description to simulate their linear and nonlinear optical response [47,53]. We approximate their electronic structure using a nearest-neighbors tightbinding model $[42,43]$. The resulting one-electron wave 

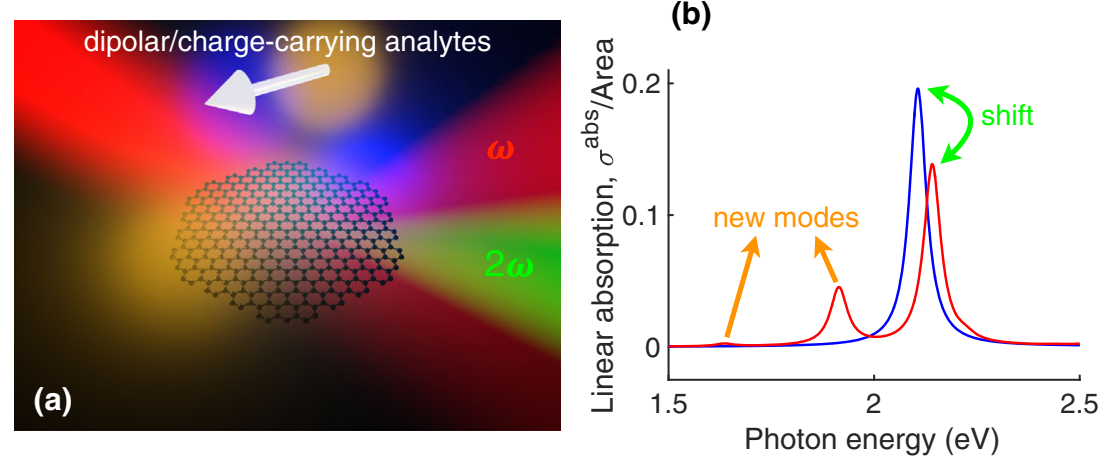

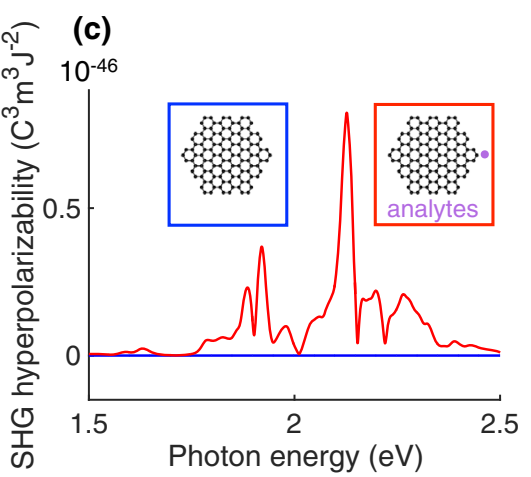

FIG. 1. Linear and nonlinear optical sensing with nanographene. (a) Illustration of an armchair-edged graphene nanohexagon (GNH) interacting with a charge- or dipole-carrying analyte (the white arrow indicates a permanent dipole moment). The molecule induces asymmetry in the nanohexagon conduction electron distribution, which can be detected by measuring either changes in the optical absorption spectrum or the onset of a second-harmonic signal. (b),(c) Linear absorption cross section (b) and second-harmonic polarizability (c) of the GNH in the absence (blue curves) or presence (red curves) of a nearby charge-carrying molecule. The analyte produces new resonance features and spectral shifts in the linear response. Additionally, it enables a large second-harmonic response from the nanohexagon, otherwise prevented by symmetry in its absence. Calculations in (b) and (c) correspond to an undoped GNH of $1 \mathrm{~nm}$ side length under the conditions of Figs. 2(a) and 4(a) for the linear and SHG responses, respectively.

functions are inserted into the random-phase-approximation susceptibility [54,55] to yield the linear response of the GNHs [53]. A straightforward generalization of this meanfield approach is followed to obtain the SHG response [47]. The interaction with the static charge or dipole of the analyte is included as an external potential, which adds up to the Hartree potential (neglecting exchange). The latter is found self-consistently following a customary iterative procedure that yields the ground state of the nanoisland in the presence of the analyte. We include inelastic processes in the response through a phenomenological relaxation lifetime $\tau$, assuming a conservative value $\tau=13$ fs (i.e., $\hbar \tau^{-1}=50 \mathrm{meV}$ ). More details on the theory are offered in the Supplemental Material (SM [56]).

The charge-carrier distribution of a graphene nanostructure can be significantly altered by the presence of a neighboring charge or dipole [51], even when the structure is electrically neutral (i.e., undoped). In particular, a chargeor dipole-carrying molecule can produce this effect [Fig. 1(a)]. The resulting modifications in the plasmonic response emerge as significant changes in the linear absorption spectrum triggered by the presence of these types of analytes [Fig. 1(b)], thus providing the basis for an optical sensor. We illustrate this effect in Fig. 1(b), which shows the absorption spectra of a neutral GNH with and without a neighboring molecule carrying an elementary charge (see below for more details). In this example, the analyte induces a blue shift of the prominent absorption peak and creates new features at lower photon energies. Even more dramatic changes are produced in the nonlinear response, which we show in Fig. 1(c) by examining the SHG nonlinear polarizability of the GNH. For the bare nanoisland (blue curve), SHG is strictly forbidden by inversion symmetry [52], while in the presence of the analyte (red curve), the broken symmetry produces intense
SHG for fundamental frequencies near the plasmon resonances of Fig. 1(b).

A more detailed analysis is presented in Fig. 2, which shows absorption cross section spectra of GNHs in the presence of a charge-carrying analyte, represented by an elementary charge $\left(Q^{\text {ext }}=e\right)$ placed at a distance $d_{x}$ from the graphene edge. The external charge produces static changes in the valence electron density (Fig. 2, top insets), which lead to modifications in the optical response (see Fig. S1 in the SM [56]). Specifically, in an undoped GNH of side length $L=1 \mathrm{~nm}$, with the external charge located $d_{x}=0.5 \mathrm{~nm}$ away from one of the hexagon corners, the static conduction charge displays a highly asymmetric distribution [Fig. 2(a)]. This causes new plasmonic modes to appear at 1.6 and $1.9 \mathrm{eV}$, which display dipolar patterns in their associated induced-charge distributions (insets). Additionally, the dominant peak of the unexposed nanoisland $\left(d_{x} \rightarrow \infty\right)$, which also shows a dipolar pattern, undergoes a slight blue shift. Obviously, these effects are reduced in magnitude when the analyte is further away from the GNH ( $d_{x}=2 \mathrm{~nm}$ curves). When moving to a larger nanoisland $[L=2 \mathrm{~nm}$, Fig. 2(c)], we observe equally substantial spectral shifts and emerging features caused by the analyte.

When the GNH is highly doped $[Q=-4 e$, corresponding to a Fermi energy $\sim 1 \mathrm{eV}$ for $L=1 \mathrm{~nm}$, Fig. 2(b)], the initial charge carrier distribution is centrosymmetric (top inset), with charges accumulating at the hexagon edges due to Coulomb repulsion. The analyte then induces a small asymmetry, which is insignificant compared to the intrinsic doping. Consequently, only minor spectral changes are observed, even at a short GNH-molecule distance $d_{x}=0.5 \mathrm{~nm}$, which nearly disappear for $d_{x}=2 \mathrm{~nm}$. High initial doping is thus detrimental for linear-absorption sensing. Nevertheless, we have considered here singly 

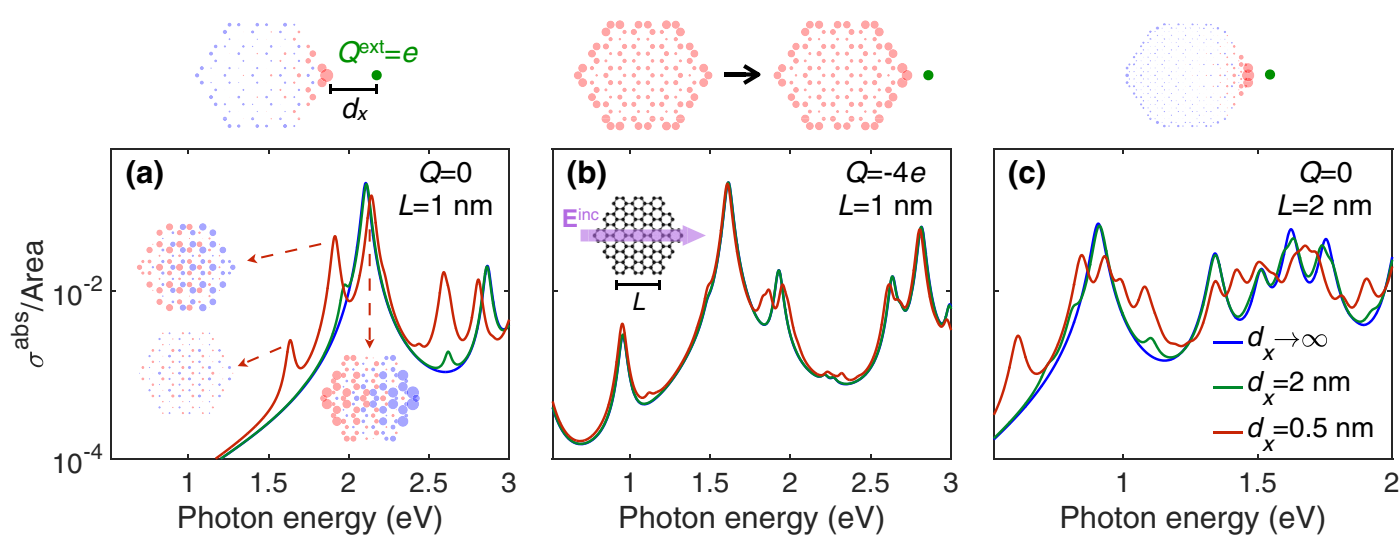

FIG. 2. Sensing of charge-carrying analytes through linear optical absorption by nanographenes. We show the linear absorption cross section of single GNHs exposed to an individual singly charged analyte $\left(Q^{\text {ext }}=e\right)$ placed in the graphene plane at different distances $d_{x}$ from the carbon edge [see legend in (c)]. Doped [net charge $Q=-4 e$, panel (b)] and undoped $[Q=0$, panels (a) and (c)] nanohexagons are considered with different side lengths $L=1$ and $2 \mathrm{~nm}$ (see labels), containing 114 and 414 carbon atoms, respectively. The incident electric field $\mathbf{E}^{\mathrm{inc}}$ is oriented as shown in the inset of (b). Conduction charge distributions are plotted above each corresponding panel for $d_{x}=0.5 \mathrm{~nm}$. Optically induced charge distributions are shown in (a) for three dominant resonances (blue and red indicate charges of opposite signs). Spectra for unexposed graphene correspond to $d_{x} \rightarrow \infty$.

charged analytes, while typical charge-carrying analytes, such as heavy metal ions, can hold many electrons, producing more intense modifications in the nanohexagon absorption, even when it is doped (see Fig. S2 in the SM [56]).

In a similar fashion, GNHs can serve as detectors for dipolar analytes, which we represent as point dipoles $\mathbf{p}^{\text {ext }}$. We note for reference that small molecules (e.g., some toxic contaminants) have typical permanent dipoles of a few Debyes [62] (e.g., $1.83 \mathrm{D}$ in hydrogen fluoride, $2.98 \mathrm{D}$ in hydrogen cyanide, and 10.6 D in hydrogen cyanide trimer). For illustration, we first consider the effect of a larger dipole $p^{\text {ext }}=50 \mathrm{D}$ (a characteristic value of $\mathrm{ZnSe}$ and CdSe nanocrystals $[63,64]$ ) placed at different distances $d_{z}$ above the hexagon center [see inset to Fig. 3(d)]; this is an academic exercise, as the size of a structure carrying such a large dipole would certainly exceed the small separation distances under consideration. We find the linear optical absorption [Figs. 3(a)-(c)] to be modified in a qualitatively similar manner as by the charged analyte (Fig. 2), with dipolar resonances appearing again in the spectra. However, when considering a smaller permanent dipole $p^{\text {ext }}=5 \mathrm{D}$ (i.e., like in the simple molecules noted above), the linear absorption is rather insensitive to the analyte, even for very small separation distances [Figs. 3(d)-(f) and Fig. S3 in the SI [56] ], so we conclude that such molecules cannot be detected in the GNH linear response.

In contrast, the GNH nonlinear response undergoes large modifications for both charge- and dipole-carrying analytes, as we show in Fig. 4, where we present secondharmonic polarizability spectra corresponding to the systems considered in Figs. 2 and 3. In the absence of the analyte $\left(d_{x} \rightarrow \infty\right)$, no signal is generated due to inversion symmetry. However, the presence of an elementary charge [Figs. 4(a)-(c)] or a dipole of 5 D [Figs. 4(d)-(f)], which are typical values for small molecules, produces a substantial
SHG response. Like in previous studies [47], this response is enhanced by plasmons of the nanostructure, particularly by the new resonances that emerge due to the GNHmolecule interaction. In fact, the values of the SHG polarizability shown in Fig. 3 are comparable to those measured for $\sim 10-20 \mathrm{~nm}$ noble-metal nanostructures [65-68], which are among the best currently available nonlinear materials. We remark that, in contrast to the insensitivity of the linear optical response to a dipole $p^{\text {ext }}=$ 5 D [Figs. 3(d)-(f)], the dipolar analyte generates an intense SHG response [Figs. 4(d)-(f)] comparable to that created by a charged analyte [Figs. 4(a)-(c)], although it quickly vanishes at distances of a few nanometers. Interestingly, the magnitude of the obtained result is roughly proportional to the inelastic relaxation time $\tau$ (see Fig. S4 in the SM [56]), a parameter that is directly affected by defects in the graphene nanoisland, and for which we use a very moderate value ( $\tau \approx 13 \mathrm{fs}$ ), well below what has been experimentally reported $[38,69]$.

In summary, we have demonstrated through atomistic quantum-mechanical simulations that nanographenes can serve as optical sensors of charge- and dipole-carrying analytes with single-molecule sensitivity. We find substantial changes in the optical absorption spectra induced by the presence of the analytes. Additionally, a strong SHG signal is enabled even in centrosymmetric nanographenes, whose second-harmonic response disappears in the absence of the external perturbation produced by the analyte. The remarkably high sensitivity that we observe in these nanostructures is inherited from the intrinsically large electro-optic tunability of graphene, which offers an extra degree of control to optimize the sensing capability. For comparison, nanographenes present a much higher SHG response than nanoparticles [47] or quantum dots [57,58] of similar size (see SM [56]). 


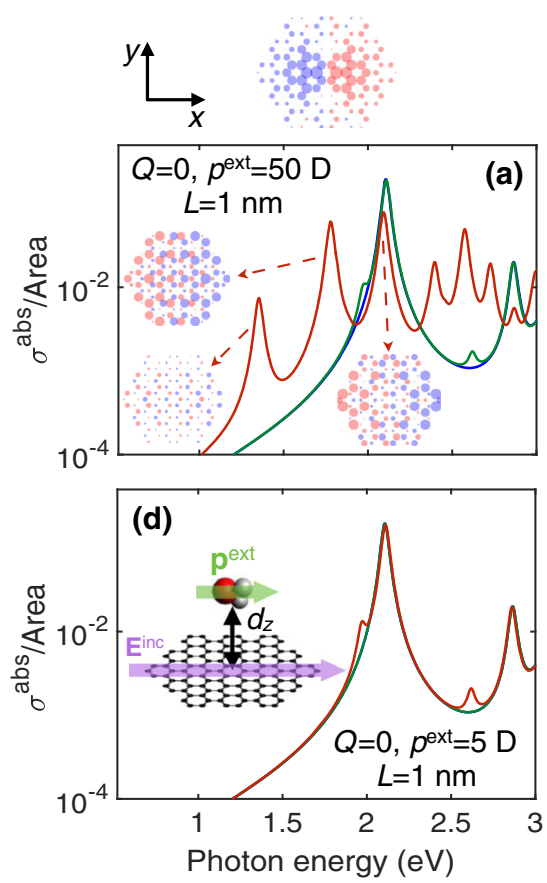

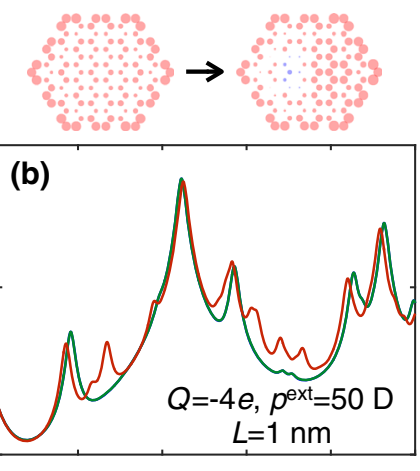

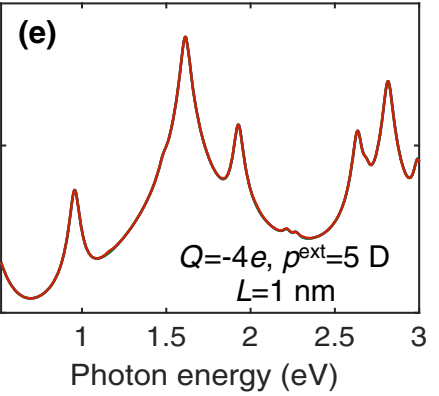

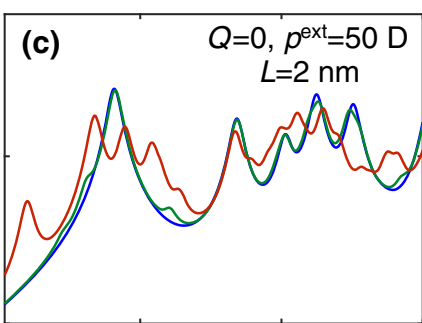

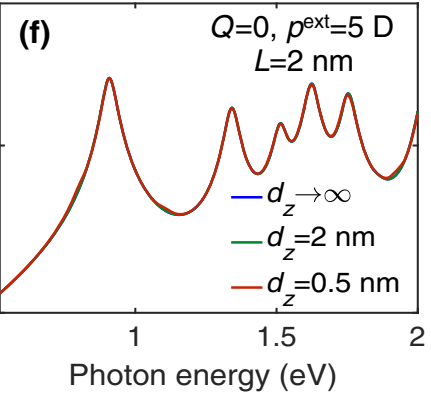

FIG. 3. Sensing of dipolar molecules through the linear optical absorption of nanographenes. We show the linear absorption cross section of individual GNHs exposed to a single analyte carrying a permanent dipole $\left[p^{\text {ext }}=50 \mathrm{D}\right.$ in (a)-(c); $p^{\text {ext }}=5 \mathrm{D}$ in (d)-(f); aligned along $x$ in all cases] placed at different distances $d_{z}$ above the hexagon center [see inset to (d)]. Doped [net charge $Q=-4 e$, panels (b) and (e)] and undoped ( $Q=0$, rest of the panels) nanohexagons are considered with different side lengths $L$, as indicated by labels. Conduction charge distributions are plotted above (a)-(c) for $d_{z}=0.5 \mathrm{~nm}$ and $p^{\text {ext }}=50$ D. Optically induced charge distributions are shown in (a) for three dominant resonances. Unexposed graphene is represented by $d_{z} \rightarrow \infty$.

As a practical realization, nanographene structures could be passivated with thin insulating layers, which would protect them from undesired chemical interactions (e.g., charge transfer) in the complex environment of the analyte. For example, nanographenes could be deposited on a dielectric substrate, and then covered with a subnanometer passivating layer (e.g., an hBN atomic film) that enables large graphene-analyte electrostatic interactions as considered in this work. Colloidal dispersions of nanographenes constitute another option, for which electrical doping produced by charge transfer from the analytes or other surrounding molecules should be taken into consideration. Incidentally, while the averaging effect due to the random spatial distribution of the analytes relative to the GNHs is
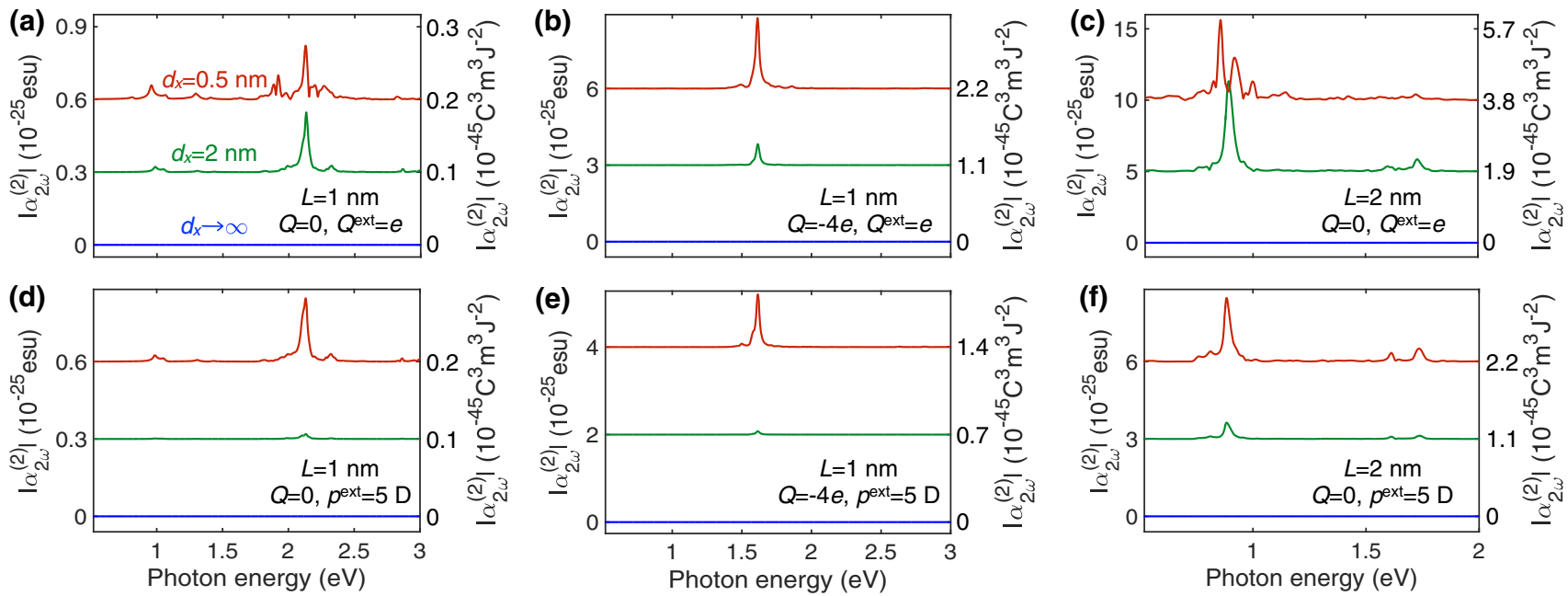

FIG. 4. Nonlinear sensing of charged and dipolar analytes. We show second-harmonic polarizability spectra of single GNHs in the presence of an individual singly charged (a)-(c) or dipole-carrying (d)-(f) molecule. Results for charged (dipolar) analytes are obtained using the same parameters as in Figs. 2(a)-(c) [Figs. 3(d)-(f)]. 
highly detrimental for linear sensing, this is not the case in nonlinear sensing (see SM [56]). It is worth mentioning that the ability to detect charge- or dipole-carrying analytes, such as hydrogen cyanide ( $\mathrm{HCN})$ or heavy metal ions, which are extremely toxic substances, is crucial for the protection of human health and the natural environment. The method explored here constitutes an attractive avenue in this direction. We analyze $\mathrm{HCN}$ in more detail in the SI [56], demonstrating that the approximation of a single point dipole compares extremely well with a more realistic treatment of the charge distribution in the molecule by natural population analysis [59]. It should be noted that an advantage of SHG sensing lies in its robustness against undesired electrical doping and variations in size and morphology of the nanographenes, provided they are centrosymmetric. We remark that the present results rely on very conservative values of the inelastic damping, and therefore, higher-quality graphene nanostructures should contribute to enhance and sharpen the optical linear and nonlinear signals. Overall, the scheme proposed here constitutes a radical departure from existing optical sensing approaches and holds great potential for pushing the limits of detectability well beyond what is currently achievable.

This work has been supported in part by the Spanish MINECO (Grants No. MAT2014-59096-P and No. SEV2015-0522), Fundació Privada Cellex, and the European Commission (CNECT-ICT-604391 and FP7ICT-2013-613024-GRASP).

*Corresponding author. javier.garciadeabajo@icfo.es

[1] K. M. Mayer, S. Lee, H. Liao, B. C. Rostro, A. Fuentes, P. T. Scully, C. L. Nehl, and J.H. Hafner, ACS Nano 2, 687 (2008).

[2] S. Lee, K. M. Mayer, and J. H. Hafner, Anal. Chem. 81, 4450 (2009).

[3] K. M. Mayer, F. Hao, S. Lee, P. Nordlander, and J. H. Hafner, Nanotechnology 21, 255503 (2010).

[4] C.-S. Cheng, Y.-Q. Chen, and C.-J. Lu, Talanta 73, 358 (2007).

[5] K.-J. Chen and C.-J. Lu, Talanta 81, 1670 (2010).

[6] M. Nuopponen and H. Tenhu, Langmuir 23, 5352 (2007).

[7] N. H. Mack, J. W. Wackerly, V. Malyarchuk, J. A. Rogers, J. S. Moore, and R. G. Nuzzo, Nano Lett. 7, 733 (2007).

[8] K. Kneipp, Y. Wang, H. Kneipp, L. T. Perelman, I. Itzkan, R. R. Dasari, and M. S. Feld, Phys. Rev. Lett. 78, 1667 (1997).

[9] S. Nie and S. R. Emory, Science 275, 1102 (1997).

[10] H. Xu, E. J. Bjerneld, M. Käll, and L. Börjesson, Phys. Rev. Lett. 83, 4357 (1999).

[11] M. Moskovits, J. Raman Spectrosc. 36, 485 (2005).

[12] J. N. Anker, W. P. Hall, O. Lyandres, N. C. Shah, J. Zhao, and R. P. Van Duyne, Nat. Mater. 7, 442 (2008).

[13] L. Rodríguez-Lorenzo, R. A. Álvarez-Puebla, I. PastorizaSantos, S. Mazzucco, O. Stéphan, M. Kociak, L. M. Liz-
Marzán, and F. J. García de Abajo, J. Am. Chem. Soc. 131, 4616 (2009).

[14] M. Lippitz, M. A. van Dijk, and M. Orrit, Nano Lett. 5, 799 (2005).

[15] M. Danckwerts and L. Novotny, Phys. Rev. Lett. 98, 026104 (2007).

[16] O. Schwartz and D. Oron, Nano Lett. 9, 4093 (2009).

[17] J. Butet, J. Duboisset, G. Bachelier, I. Russier-Antoine, E. Benichou, C. Jonin, and P.-F. Brevet, Nano Lett. 10, 1717 (2010).

[18] J. Butet, G. Bachelier, I. Russier-Antoine, C. Jonin, E. Benichou, and P.-F. Brevet, Phys. Rev. Lett. 105, 077401 (2010).

[19] H. Harutyunyan, G. Volpe, R. Quidant, and L. Novotny, Phys. Rev. Lett. 108, 217403 (2012).

[20] Y. Kim, R. C. Johnson, and J. T. Hupp, Nano Lett. 1, 165 (2001).

[21] A. K. Singh, D. Senapati, S. Wang, J. Griffin, A. Neely, P. Candice, K. M. Naylor, B. Varisli, J. R. Kalluri, and P. C. Ray, ACS Nano 3, 1906 (2009).

[22] A. Neely, C. Perry, B. Varisli, A. K. Singh, T. Arbneshi, D. Senapati, J. R. Kalluri, and P. C. Ray, ACS Nano 3, 2834 (2009).

[23] M. Mesch, B. Metzger, M. Hentschel, and H. Giessen, Nano Lett. 16, 3155 (2016).

[24] B. Wunsch, T. Stauber, F. Sols, and F. Guinea, New J. Phys. 8, 318 (2006).

[25] E. H. Hwang and S. Das Sarma, Phys. Rev. B 75, 205418 (2007).

[26] M. Jablan, H. Buljan, and M. Soljačić, Phys. Rev. B 80, 245435 (2009).

[27] L. Ju, B. Geng, J. Horng, C. Girit, M. Martin, Z. Hao, H. A. Bechtel, X. Liang, A. Zettl, Y. R. Shen et al., Nat. Nanotechnol. 6, 630 (2011).

[28] F. H. L. Koppens, D. E. Chang, and F. J. García de Abajo, Nano Lett. 11, 3370 (2011).

[29] J. Chen, M. Badioli, P. Alonso-González, S. Thongrattanasiri, F. Huth, J. Osmond, M. Spasenović, A. Centeno, A. Pesquera, P. Godignon et al., Nature (London) 487, 77 (2012).

[30] Z. Fei, A. S. Rodin, G. O. Andreev, W. Bao, A. S. McLeod, M. Wagner, L. M. Zhang, Z. Zhao, M. Thiemens, G. Dominguez et al., Nature (London) 487, 82 (2012).

[31] H. Yan, X. Li, B. Chandra, G. Tulevski, Y. Wu, M. Freitag, W. Zhu, P. Avouris, and F. Xia, Nat. Nanotechnol. 7, 330 (2012).

[32] H. Yan, Z. Li, X. Li, W. Zhu, P. Avouris, and F. Xia, Nano Lett. 12, 3766 (2012).

[33] Z. Fang, S. Thongrattanasiri, A. Schlather, Z. Liu, L. Ma, Y. Wang, P. M. Ajayan, P. Nordlander, N. J. Halas, and F. J. García de Abajo, ACS Nano 7, 2388 (2013).

[34] V. W. Brar, M. S. Jang, M. Sherrott, J. J. Lopez, and H. A. Atwater, Nano Lett. 13, 2541 (2013).

[35] R. Alaee, M. Farhat, C. Rockstuhl, and F. Lederer, Opt. Express 20, 28017 (2012).

[36] R. Yu, R. Alaee, F. Lederer, and C. Rockstuhl, Phys. Rev. B 90, 085409 (2014).

[37] F. J. García de Abajo, ACS Photonics 1, 135 (2014).

[38] D. Rodrigo, O. Limaj, D. Janner, D. Etezadi, F. J. García de Abajo, V. Pruneri, and H. Altug, Science 349, 165 (2015).

[39] L. Wu, H. S. Chu, W. S. Koh, and E. P. Li, Opt. Express 18, 14395 (2010). 
[40] Y. Li, H. Yan, D. B. Farmer, X. Meng, W. Zhu, R. M. Osgood, T. F. Heinz, and P. Avouris, Nano Lett. 14, 1573 (2014).

[41] A. Marini, I. Silveiro, and F. J. García de Abajo, ACS Photonics 2, 876 (2015).

[42] P. R. Wallace, Phys. Rev. 71, 622 (1947).

[43] A. H. Castro Neto, F. Guinea, N. M. R. Peres, K. S. Novoselov, and A. K. Geim, Rev. Mod. Phys. 81, 109 (2009).

[44] S. A. Mikhailov, Europhys. Lett. 79, 27002 (2007).

[45] E. Hendry, P. J. Hale, J. Moger, A. K. Savchenko, and S. A. Mikhailov, Phys. Rev. Lett. 105, 097401 (2010).

[46] T. J. Constant, S. M. Hornett, D. E. Chang, and E. Hendry, Nat. Phys. 12, 124 (2016).

[47] J. D. Cox and F. J. García de Abajo, Nat. Commun. 5, 5725 (2014).

[48] J. D. Cox, I. Silveiro, and F. J. García de Abajo, ACS Nano 10, 1995 (2016).

[49] X. Yan, X. Cui, and L. shi Li, J. Am. Chem. Soc. 132, 5944 (2010).

[50] K. Müllen, ACS Nano 8, 6531 (2014).

[51] I. Silveiro and F. J. García de Abajo, Appl. Phys. Lett. 104, 131103 (2014).

[52] R. W. Boyd, Nonlinear Optics, 3rd ed. (Academic Press, Amsterdam, 2008).

[53] S. Thongrattanasiri, A. Manjavacas, and F. J. García de Abajo, ACS Nano 6, 1766 (2012).

[54] D. Pines and P. Nozières, The Theory of Quantum Liquids (W. A. Benjamin, Inc., New York, 1966).

[55] L. Hedin and S. Lundqvist, in Solid State Physics, edited by F. Seitz and H. Ehrenreich (Academic Press, New York, 1970), Vol. 23, pp. 1-181.

[56] See Supplemental Material at http://link.aps.org/ supplemental/10.1103/PhysRevLett.117.123904 for details of the quantum mechanical simulation of the optical response of nanographenes, a comparison of nonlinear sensing with quantum dots and with metal nanoparticles, and further simulation results. This material includes additional Refs. [57-61].

[57] D. Petrov, B. Santos, G. Pereira, and C. de Mello Donegá, J. Phys. Chem. B 106, 5325 (2002).

[58] Y. Zhang, M. Ma, X. Wang, D. Fu, H. Zhang, N. Gu, J. Liu, Z. Lu, L. Xu, and K. Chen, J. Phys. Chem. Sol. 64, 927 (2003).

[59] R. Arnaud, C. Adamo, M. Cossi, A. Milet, Y. Vallée, and V. Barone, J. Am. Chem. Soc. 122, 324 (2000).

[60] Y. Zhang, X. Wang, D. Fu, J. Cheng, Y. Shen, J. Liu, and Z. Lu, J. Phys. Chem. Sol. 62, 903 (2001).

[61] Y. Zhang, M. Ma, X. Wang, D. Fu, N. Gu, J. Liu, Z. Lu, Y. Ma, L. Xu, and K. Chen, J. Phys. Chem. Sol. 63, 2115 (2002).

[62] W. M. Haynes, CRC Handbook of Chemistry and Physics (CRC Press, London, 2014).

[63] B. Dabbousi, J. Rodriguez-Viejo, F. V. Mikulec, J. Heine, H. Mattoussi, R. Ober, K. Jensen, and M. Bawendi, J. Phys. Chem. B 101, 9463 (1997).

[64] M. Shim and P. Guyot-Sionnest, J. Chem. Phys. 111, 6955 (1999).

[65] I. Russier-Antoine, E. Benichou, G. Bachelier, C. Jonin, and P. F. Brevet, J. Phys. Chem. C 111, 9044 (2007).

[66] P. Galletto, P. F. Brevet, H. H. Girault, R. Antoine, and M. Broyer, Chem. Commun. 581 (1999).

[67] I. Russier-Antoine, J. Duboisset, G. Bachelier, E. Benichou, C. Jonin, N. D. Fatti, F. Vallée, A. Sánchez-Iglesias, I. Pastoriza-Santos, L. M. Liz-Marzán et al., J. Phys. Chem. Lett. 1, 874 (2010).

[68] A. K. Singh, D. Senapati, A. Neely, G. Kolawole, C. Hawker, and P. C. Ray, Chem. Phys. Lett. 481, 94 (2009).

[69] P. Tassin, T. Koschny, and C. M. Soukoulis, Science 341, 620 (2013). 